\title{
New lanthanide phosphonates structures obtained using XRPD data
}

\author{
E. Fernández-Zapico ${ }^{1, *}$, J. Montejo-Bernardo ${ }^{1}$, \\ S. García-Granda ${ }^{1}$, J. R. García ${ }^{1}$, G. R. Castro ${ }^{2}$, \\ F. Y. Liu ${ }^{3}$, J. Rocha ${ }^{3}$
}

${ }^{1}$ Departments of Physical and Analytical Chemistry and Organic and Inorganic Chemistry, University of Oviedo-CINN, Spain

${ }^{2}$ SpLine, Spanish CRG Beamline, ESRF, BP220, 38043 Grenoble Cedex, France

${ }^{3}$ Department of Chemistry, CICECO, University of Aveiro, Portugal

*Contact author; e-mail: fernandezeva.uo@uniovi.es

Keywords: lanthanide phosphonates, X-ray diffraction, Rietveld refinement

\begin{abstract}
Seven lanthanide diphosphonates, $\left[\mathrm{H}_{3} \mathrm{~N}\left(\mathrm{CH}_{2}\right)_{4} \mathrm{NH}_{3}\right] \mathrm{Ln}\left[\right.$ hedpH] $\left[h_{\text {hedpH}}\right](\mathrm{Ln}=\mathrm{La}$, Pr, Sm, Eu, Gd, Tb, Er; hedp = 1-hydroxyethylidenediphosphonate) have been synthesized with 1,4-diaminobutane as the template. The structures were obtained starting from the known X-ray single crystal model of lanthanum compound, with the X-ray powder diffraction data for these seven compounds. H-atoms were introduced using geometrical considerations. Rietveld fits of the experimental diffractograms confirm the isostructurality of all compounds in the series, and show the different behaviour between the two distances M-M existing in the structures.
\end{abstract}

\section{Introduction}

Metal phosphonate chemistry is of great interest in material science due to their potential applications in many fields such as ion-exchange, catalysis, and sensor devices [1,2]. Often, metal phosphonates exhibit polymeric structures consisting of chains, layers, or threedimensional networks, but monomeric structures are also known. In particular, the tunable organic units in diphosphonate $\left[\mathrm{R}\left(\mathrm{PO}_{3}\right)_{2}{ }^{4-}\right]$ allow the construction of metal phosphonate materials with new architectures, including open framework structures $[3,4]$ and porous pillared layered structures $[5,6]$.

Components of the family $\left[\mathrm{H}_{3} \mathrm{~N}\left(\mathrm{CH}_{2}\right)_{4} \mathrm{NH}_{3}\right] \mathrm{Ln}\left[\right.$ hedpH] $\left[\right.$ hedpH $\left.\mathrm{H}_{2}\right]$, with $\mathrm{Ln}=\mathrm{La}, \mathrm{Pr}, \mathrm{Sm}, \mathrm{Tb}$ and Er has been recently reported by our research group [7,8] and the crystal structure of the La compound was already published by Liu and collaborators [9]. Following with the work previously developed [10] we present here the results of the structural study for seven components of the family ( $\mathrm{Ln}=\mathrm{La}, \mathrm{Pr}, \mathrm{Sm}, \mathrm{Eu}, \mathrm{Gd}, \mathrm{Tb}$ and $\mathrm{Er}$ ) using their synchrotron XRPD data and the La X-ray single crystal data. 


\section{X-ray crystallographic analysis}

\subsection{Data collection}

X-ray single crystal data were collected in the laboratory diffractometer Nonius Kappa CCD, using $\mathrm{Cu}-\mathrm{K}_{\alpha}$ radiation $(\lambda=1.5418 \AA)$, at room temperature. The powder data were collected at the ESRF (BM25-Spline, $\lambda=0.8290419 \AA$ ), in a range between 3.5 and $60^{\circ}$, with a step size of $0.02^{\circ}$ at room temperature.

\subsection{Structural refinement}

In a Rietveld refinement with the FullProf program [11], unit cell parameters and the positions of the non H-atoms were refined, being the position of the H-atoms completed using the program Mercury [12], taking into account geometrical considerations, as is usually done in the case of single crystal data. The final structural results show the isostructurality of the seven compounds, tuned by the influence of the cations.

For the Rietveld refinement, the single crystal structure of lanthanum compound [9] was used as initial model in all cases.

The XRPD profiles have been modeled as a pseudo-Voigt function. We have refined the zero offset, the scale factor, six background terms, the $\eta$ parameter, the $\mathrm{W}$ Caglioti parameter, the unit cell, and the position of all atoms, excluding all hydrogens and template atoms, except in the case of terbium compound, in which only the coordinates of the central atom and the oxygens around it were refined. The refinement was made in the range $2 \theta=3.5-45.0^{\circ}$.

\section{Results and discussion}

The refinement indexes of agreement for the seven structures analyzed are listed in table 1. Very low values are reached for all the cases, except for the terbium compound, due to the low quality of the XRPD data and the small number of atomic parameters that could be refined. Even so, all values obtained for the adjustment figures of merit are within acceptable ranges [13]. As an example, the final Rietveld refinement for the Eu compound is showed in figure 1 (all the others are closely similar to this). We can observe that the model adjusts very well with the experimental values

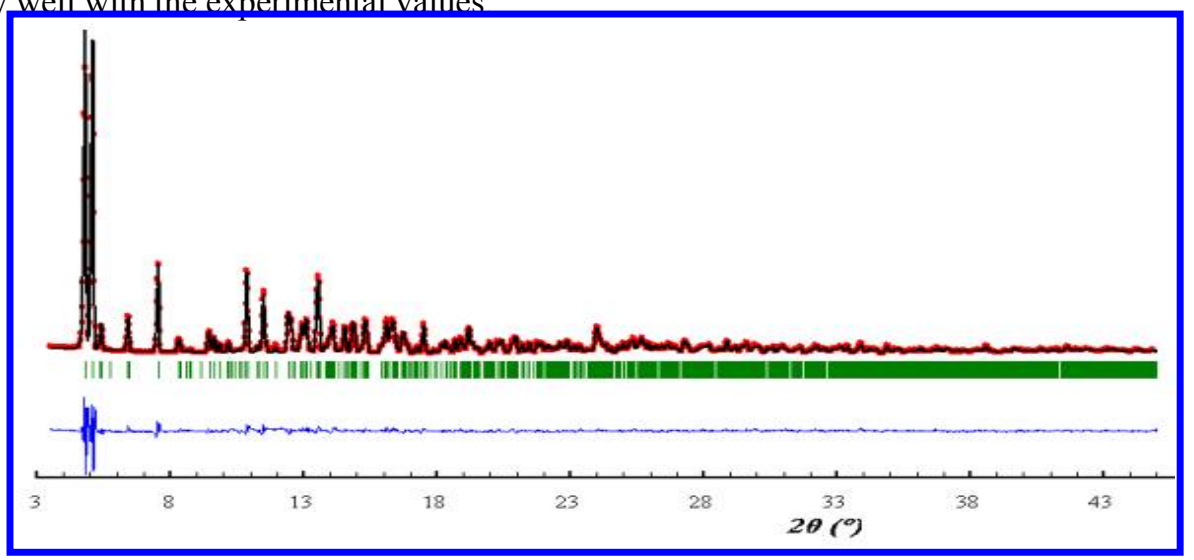

Figure 1. Rietveld refinement for the Eu compound. 
Table 1. Refinement indexes of agreement for the analyzed compounds.

\begin{tabular}{|c|c|c|c|c|c|c|c|}
\hline $\boldsymbol{L n}$ & La & Pr & Sm & Eu & Gd & Tb & Er \\
\hline $\boldsymbol{R}_{\boldsymbol{B}}$ & 6.50 & 5.53 & 4.76 & 4.82 & 4.36 & 8.81 & 5.39 \\
\hline $\boldsymbol{R}_{\boldsymbol{F}}$ & 5.11 & 4.73 & 4.22 & 4.01 & 3.77 & 8.00 & 4.92 \\
\hline
\end{tabular}

As explained in the description of the La compound [9], due to the different binding mode of phosphonates, it can be defined two distinct faces in the polyhedra $\mathrm{LaO}_{7}$, related to its opposite by an inversion center (see figure 2). This causes alternating short and long Ln-Ln distances, as shown in table 2 , which vary in a defined and different way with the metal radius. Long distances remain almost constant, with a maximum variation of $0.06 \AA(\approx 1 \%)$, and short distances decrease $(0.32 \AA)$ with decreasing size of the central atom. This shortening is due to the decrease of the bond's distances with the coordinated oxygens $\mathrm{O}(1)$ and $\mathrm{O}(6)$, and the same goes for the angle $\mathrm{O}(2)-\mathrm{P}(2)-\mathrm{O}(6)$, involved in the Ln-Ln connection. No significant changes were observed for the rest of the $\mathrm{M}-\mathrm{O}$ distances and O-P-O angles by varying the central atom.

Table 2. Ln-Ln distances (from Rietveld fit).

\begin{tabular}{|c|c|c|}
\hline Ln & $\boldsymbol{d}($ short $) / \boldsymbol{A}$ & $\boldsymbol{d}($ long $) / \boldsymbol{A}$ \\
\hline $\mathbf{L a}$ & $4.9281(5)$ & $5.8451(5)$ \\
\hline $\mathbf{P r}$ & $4.816(2)$ & $5.872(3)$ \\
\hline $\mathbf{S m}$ & $4.735(2)$ & $5.897(3)$ \\
\hline $\mathbf{E u}$ & $4.729(2)$ & $5.904(3)$ \\
\hline $\mathbf{G d}$ & $4.7098(1)$ & $5.8991(2)$ \\
\hline $\mathbf{T b}$ & $4.6084(1)$ & $5.8430(2)$ \\
\hline $\mathbf{E r}$ & $4.6168(4)$ & $5.8937(5)$ \\
\hline
\end{tabular}

Looking at the changes on the unit cell volume with reference to the Shannon's effective ionic radii [14], we observe the expected trend: as we move towards the right in the periodic table the radius decreases linearly, and so does the volume of the cell. Table 3 shows the values of unit cell volumes and Shannon's effective ionic radii for the seven studied compounds and, in table 4, cell parameters after Rietveld refinement for all compounds are listed. 


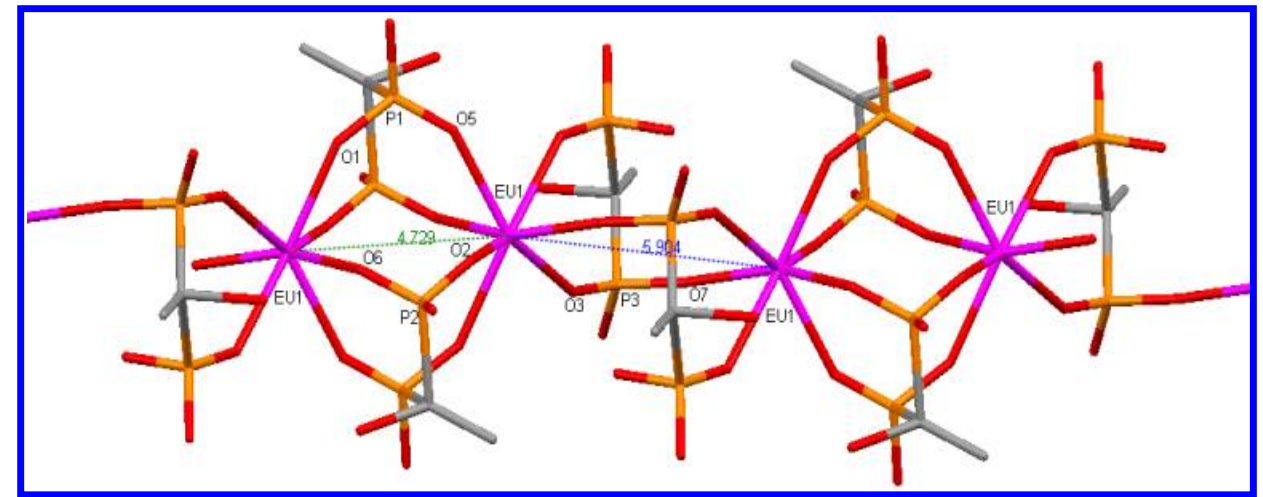

Figure 2. Ln-Ln distances for the Eu compound.

Table 3. Shannon's ionic radii and unit cell volume after refinement.

\begin{tabular}{|c|c|c|}
\hline Ln & $\boldsymbol{R}_{\text {Shannon }} / \boldsymbol{A}$ & $\boldsymbol{V}_{\text {cell }} / \boldsymbol{A}^{\mathbf{3}}$ \\
\hline $\mathbf{L a}$ & 1.10 & $1000.23(9)$ \\
\hline $\mathbf{P r}$ & 1.06 & $992.56(9)$ \\
\hline $\mathbf{S m}$ & 1.02 & $985.67(8)$ \\
\hline $\mathbf{E u}$ & 1.01 & $986.77(9)$ \\
\hline $\mathbf{G d}$ & 1.00 & $983.37(9)$ \\
\hline $\mathbf{T b}$ & 0.98 & $961.8(1)$ \\
\hline $\mathbf{E r}$ & 0.95 & $972.16(9)$ \\
\hline
\end{tabular}

Table 4. Cell parameters after Rietveld refinement for the seven compounds.

\begin{tabular}{|c|c|c|c|c|c|c|}
\hline $\boldsymbol{L n}$ & $\boldsymbol{a}(\boldsymbol{A})$ & $\boldsymbol{b}(\boldsymbol{A})$ & $\boldsymbol{c}(\boldsymbol{A})$ & $\boldsymbol{\alpha}\left({ }^{\circ}\right)$ & $\boldsymbol{\beta}\left({ }^{\circ}\right)$ & $\boldsymbol{\gamma}\left({ }^{\circ}\right)$ \\
\hline $\boldsymbol{L a}$ & $10.2287(6)$ & $10.7286(6)$ & $10.7395(6)$ & $67.446(4)$ & $90.024(5)$ & $68.655(4)$ \\
\hline $\boldsymbol{P r}$ & $10.1795(5)$ & $10.6473(6)$ & $10.8527(6)$ & $66.691(4)$ & $89.564(5)$ & $68.566(4)$ \\
\hline $\boldsymbol{S} \boldsymbol{m}$ & $10.1438(5)$ & $10.5911(5)$ & $10.8908(6)$ & $66.419(4)$ & $89.380(4)$ & $68.583(3)$ \\
\hline $\boldsymbol{E} \boldsymbol{u}$ & $10.1517(5)$ & $10.5882(5)$ & $10.9284(6)$ & $66.104(3)$ & $89.294(4)$ & $68.547(4)$ \\
\hline $\boldsymbol{G d}$ & $10.1335(5)$ & $10.5598(6)$ & $10.9399(6)$ & $66.090(4)$ & $89.166(5)$ & $68.510(4)$ \\
\hline $\boldsymbol{T} \boldsymbol{b}$ & $10.0580(7)$ & $10.4041(8)$ & $10.9572(8)$ & $65.807(5)$ & $88.785(7)$ & $68.491(5)$ \\
\hline $\boldsymbol{E} \boldsymbol{r}$ & $10.0955(5)$ & $10.4574(5)$ & $10.9855(6)$ & $65.785(4)$ & $88.753(5)$ & $68.426(4)$ \\
\hline
\end{tabular}




\section{Concluding remarks}

The Rietveld refinements of XRPD data for seven members of $\left[\mathrm{H}_{3} \mathrm{~N}\left(\mathrm{CH}_{2}\right)_{4} \mathrm{NH}_{3}\right] \mathrm{Ln}\left[\right.$ hedpH] $\left[\right.$ hedpH $\left.\mathrm{H}_{2}\right]$ family $(\mathrm{Ln}=\mathrm{La}, \mathrm{Pr}, \mathrm{Sm}, \mathrm{Eu}, \mathrm{Gd}, \mathrm{Tb}$ and $\mathrm{Er})$ have been performed, confirming the isostructurality of all compound, and showing the different behavior between the two existing Ln-Ln distances in the structures, related to the bond's distances of the central atom with its coordinated oxygens, and the relationship between the unit cell volume and the metal in the complexes.

\section{References}

1. Clearfield, A, 1996, Curr. Opin. Solid State Mater. Sci., 1, 268-278.

2. Clearfield, A, 2002, Curr. Opin. Solid State Mater. Sci., 6, 495-506.

3. Serpaggi, F. \& Férey, G., 1999, Microporous Mesoporous Mater., 32, 311-318.

4. Riou, D., Belier, F., Serre, C., Nogues, M., Vichard, D. \& Férey, G., 2000, Int. J. Inorg. Mater., 2, 29-33.

5. Serpaggi, F. \& Férey, G., 1998, Inorg. Chem., 38, 4741-4744.

6. Gómez-Alcántara, M.M., Cabeza, A., Moreno-Real, L., Aranda, M.A.G. \& Clearfield, A., 2006, Microporous Mesoporous Mater., 88, 293-303.

7. Roces, L., García-Granda, S., García, J.R., Liu, F.Y. \& Carlos, L.D., 2006, Acta Crystallogr., A62, S268.

8. Fernández-Zapico, E., Roces, L., García-Granda, S., García, J.R., Liu, F.Y. \& Rocha, J., 2007, Acta Crystallogr., A63, S183.

9. Liu, F.Y., Roces, L., Sa Ferreira, R.A., García-Granda, S., García, J.R., Carlos, L.D. \& Rocha, J., 2007, J. Mater. Chem., 17, 3696-3701.

10. Fernández-Zapico, E., Montejo, J., Roces, L., García-Granda, S., Khainakov, S.A., García, J.R., Liu, F.Y. \& Rocha, J., 2009, Acta Crystallogr., A65, S319-320.

11. Rodríguez-Carvajal, J., 1993, Physica B., 192, 55.

12. Macrae, C.F., Bruno, I.J., Chisholm, J.A., Edgington, P.R., McCabe, P., Pidcock, E., Rodriguez-Monge, L., Taylor, R., van de Streek, J. \& Wood, P.A., 2008, J. Appl. Crystallogr., 41, 466-470.

13. Toby, B.H., 2006, Powder Diffr., 21, 67-70.

14. Shannon, R.D., 1976, Acta Crystallogr., A32, 751-767.

Acknowledgements. Financial support from Spanish MICINN (MAT2006-01997, MAT2010-15095 and 'Factoría de Cristalización' Consolider Ingenio 2010), Universidad de Oviedo and Banco Santander is acknowledged. FEDER support is also acknowledged. 